\title{
Tempo and mode in the influence of global climatic changes on mammalian evolution.
}

\author{
Manuel Hernández Fernández ${ }^{1,2}$, Juan L. CANTALAPIEdRA ${ }^{3}$, Ana R. Gómez CANO \\ ${ }^{1}$, Blanca A. GARCÍA Yelo ${ }^{1,3}$ \& Ana MORENO BOFARULL ${ }^{1}$ \\ 1 Departamento de Paleontología, Facultad de Ciencias Geológicas, Universidad \\ Complutense de Madrid \\ ${ }^{2}$ Unidad Externa de Investigación en Paleontología, Instituto de Geología Económica, \\ Consejo Superior de Investigaciones Científicas \\ 3 Departamento de Paleobiología, Museo Nacional de Ciencias Naturales, Consejo \\ Superior de Investigaciones Científicas
}

\begin{abstract}
Due to their extraordinary fossil record during the Cenozoic, mammals are a key factor in order to understand how global climatic changes have shaped biotic evolution, especially in continental environments. During the last decades, climatic influence on the evolution of mammal faunas has been reflected mainly in two sets of studies. First, there are works focused in the use of observed variations in the structure of mammalian palaeocommunities through extensive time sequences in order to detect significant palaeoclimatic changes. These studies are based in the concepts of community convergence and uniformitarism, and make use of modern ecological analogues to infer the climatic conditions in which the extinct faunas lived. On the other hand, when data on faunal change through time are confronted with independent data on palaeoclimatic changes it may be statistically inferred whether there has been simultaneous occurrence of climatic changes, faunal turnover and modifications in the ecological structure of the palaeofaunas. The second set of studies is based on the statistical analysis of ecological data from modern faunas. Extant assemblages reflect in their ecological characteristics the past influence of global climate changes on the evolution of mammalian lineages living today, both in geographical and cladal basis. Finally, there are new works based on the study of the phylogenetic signal that can be found in modern clades, which are able to identify periods of enhanced or reduced cladogenesis. Although all these approaches cannot be used simultaneously because of latent problems derived of circular reasoning, the development of methodological protocols for the combined analysis of their results has the potential to shed new light on the question of how and when climate changes affect evolution of biotic communities.

\#
\end{abstract}

Session on "Linked effects of climate change, orbital forcing, tectonics and biotic interaction: Which model is required to explain faunal evolution during the Quaternary?” 